\title{
Digital Transformation of Human Resource Processes in Small and Medium Sized Enterprises using Robotic Process Automation
}

\author{
Cristina Elena Turcu ${ }^{1}$ \\ Computers Department \\ Stefan cel Mare University of Suceava \\ Suceava, Romania
}

\author{
Corneliu Octavian Turcu ${ }^{2}$ \\ Computers, Electronics and Automation Department \\ Stefan cel Mare University of Suceava \\ Suceava, Romania
}

\begin{abstract}
The aim of this paper was to obtain data and information on the digital transformation of human resource (HR) processes in small- and medium-sized enterprises (SMEs) with the help of robotic process automation (RPA), in order to increase competitiveness in the digital age. Romanian businesses are attempting to close the gap with companies in developed countries by implementing projects that allow the adoption of emerging technologies in HR departments. This paper presents some of the preliminary findings, resulted from a collaboration between a university and an SME, for the efficient implementation of specific HR processes using RPA. The paper provides a brief introduction of the RPA concept as well as a list of HR processes that can be automated within enterprises, with the benefits brought to the enterprise and employees presented in both qualitative and quantitative terms for each HR process. In addition, a case study for the automatic collection of candidates' documents and extraction of primary information about them was considered. Further on, the problems encountered during implementation were listed, along with potential solutions. Given the benefits offered, RPA could play an important role in transitioning HR functions into the digital era.
\end{abstract}

Keywords-Robotic process automation (RPA); small- and medium-sized enterprises (SME); human resource (HR); digital HR; recruitment

\section{INTRODUCTION}

We are currently witnessing multiple challenges for companies, such as demographic and social changes, technological advances, etc. In order to meet these challenges, companies need to be agile and adaptable. An important role in fulfilling these expectations, in the conditions of ongoing and massive disruptions, is played by human resources. The good organization of human resources allows the optimization of the employees' work, even in the conditions of the disturbances introduced by the COVID pandemic.

If before the global pandemic, "digital technologies did not find a strong and widely based application in the small- and medium-sized enterprises (SMEs) sector", "due to the COVID19 disruption, SMEs are now trying to avoid a total shut down of economic activities by introducing digital technologies" [1].

In all US industries, according to the research conducted by the McKinsey Global Institute in 2016, approximately 17\% of work consists of data collection and 16\% of data processing tasks that at the time of the study were largely performed by human workers [2].

In small- and medium-sized businesses, human resource (HR) departments typically suffer from aging IT systems. Employees of these departments often need to:

- Enter data into multiple systems;

- Toggle between different applications, entering in one application data retrieved from another one.

- Reconcile data across two or more systems.

To quickly streamline these routine tasks without upgrading or replacing existing old systems, Robotic Process Automation (RPA) technology offers solutions. Thus, much of the manual work involved in these tasks can be automated using RPA.

RPA adoption is growing every year. According to Gartner, in 2020 RPA was, for the second year in a row, the fastestgrowing segment in the enterprise software market, with a $38.9 \%$ increase to $\$ 1.9$ billion in revenues [3]. Several studies worldwide estimated an increase in the use of RPA in various fields. Thus, for example, Transforma Insights expects total market spending for RPA will increase from $\$ 1.2$ billion in 2020 to $\$ 13$ billion in 2030 [4].

Over the past few years, we are witnessing the digital HR transformation, "the application of advanced technologies and analytics, digital traits and behaviors, and HR customer centricity through the lens of the organizations HR Operating Model to optimize HR to deliver sustainable organizational performance" (Fig. 1) [5].

Analysts from McKinsey \& Company called robotic process automation technology a "third arm" for HR organizations because it works with HR to amplify the department’s capacities [6].

According to a survey conducted by $\mathrm{PwC}$ on $\mathrm{HR}$ technologies, 45\% of respondents (approx. $600 \mathrm{HR}$ and HR information technology leaders on six continents) intend to invest in hyper-automation or robotic process automation (RPA) technology in the following 12-24 months [7]. 


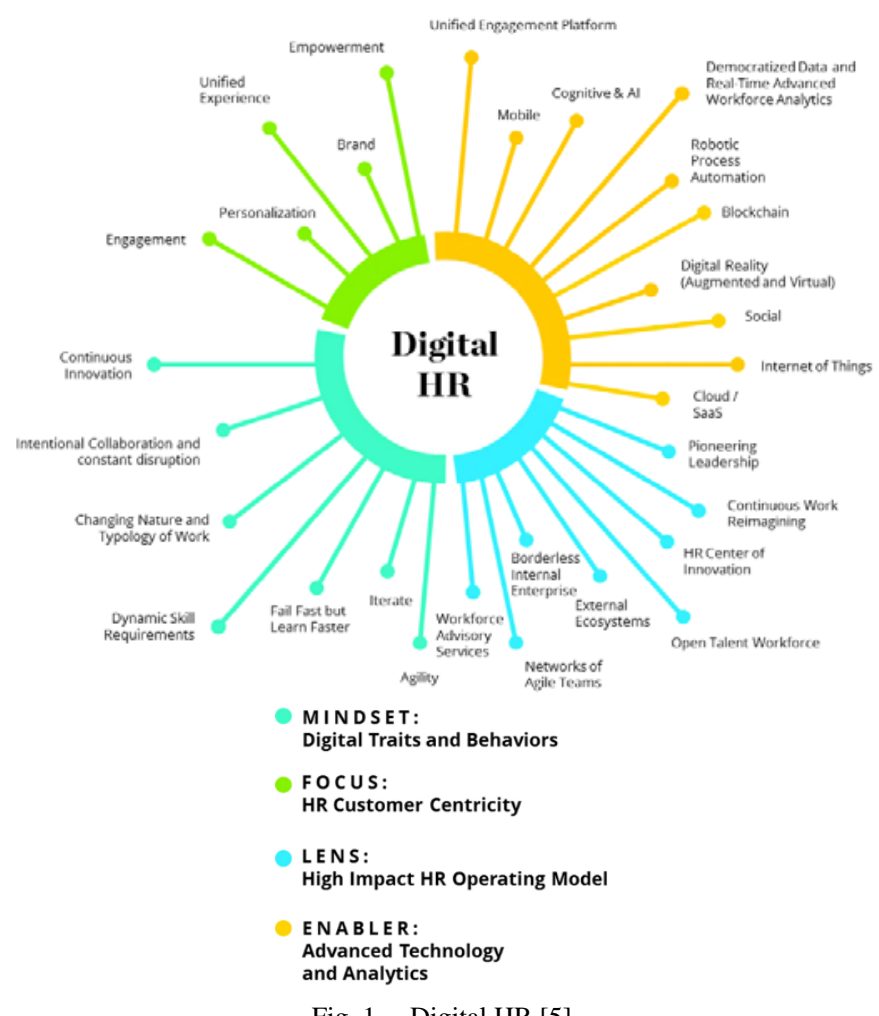

Fig. 1. Digital HR [5].

Published studies on the adoption of RPA in companies refer mainly to large companies. Small- and medium-sized enterprises are often overlooked. Our research seeks to fill a gap in the scientific literature on robotic process automation in human resource departments of SMEs, aiming to ensure the potential transfer of knowledge from academia to industry.

In this regard, we try to provide answers to the following research questions (RQ):

\section{RQ1. What is Robotic Process Automation (RPA)?}

RQ2. What are the HR processes in SMEs that can be automated with RPA?

RQ3. What advantages would the adoption of RPA bring in the HR departments of small- and medium-sized enterprises (SMEs)?

RQ4. What are the challenges HR departments are faced with when adopting RPA-based solutions?

To capture relevant knowledge on the topic, we conducted a literature review considering six of the databases frequently used by researchers: Web of Science, Springer, Science Direct, IEEE Xplorer, Scopus, and Google Scholar. In order to gain more insights for this paper, we also conducted a backwardand-forward search.

A further step in our research was the expert interviews, used to confirm and strengthen the list of use cases identified in the literature. Thus, we conducted a semi-structured in-depth interview with senior HR personnel from various SMEs. During the interviews, we considered the following topics: RPA objectives in HR, types of projects, benefits, impact on jobs, challenges. We reviewed the interview responses to obtain key insights that are summarized in the present paper.

The remainder of this paper is organized as follows. In the next section we present some definitions of the term Robotic Process Automation. Section III refers to several achievements in the field, while Section IV presents the processes of HR departments in SMEs where RPA can be incorporated along with their benefits that SMEs and employees can obtain by using RPA tools. The next section presents the flow of an application under development within a national project, mentioning some issues encountered during implementation. Further on, authors highlight the future scope of the technology, and in the last section of the paper we formulate the conclusions of our research.

\section{THEORETICAL BACKGROUND}

\section{A. Robotic Process Automation (RPA)}

In order to answer the first research question and to clarify the concept of Robotic Process Automation (RPA), we present some selected definitions that we believe are relevant to our discussion.

IEEE Corporate Advisory Group defines RPA as the use of a "preconfigured software instance that uses business rules and predefined activity choreography to complete the autonomous execution of a combination of processes, activities, transactions, and tasks in one or more unrelated software systems to deliver a result or service with human exception management.” [8].

RPA could be described as an "emerging form of business process automation technology based on the notion of software robots or artificial intelligence (AI) workers. RPA has become the new language of business. This technology is more powerful among the 21st century technologies" [9].

RPA technology is non-invasive for existing systems; it does not replace them, but interacts with them. In fact, it's a new layer above the organization's applications and cloud services that must integrate easily and efficiently with all of these.

The concept of robotic process automation is becoming increasingly integrated into various domains as a means for improving productivity, compliance, product quality, etc. In the gray literature, many key use cases are examined, presenting the experience of various organizations that have adopted RPA. The use of this technology allows the emulation of actions taken by human users, so that work processes can be automatically implemented in many business functions, such as: Human Resource, Finance \& Accounting, Production, Sales \& Marketing, Supply Chain, and Information Technology.

In this paper, authors focus on human resource departments. HR professionals deal with many processes and sub-processes, such as hiring, onboarding, employee benefits administration, managing complex workplace situations, enforcing regulatory changes. This usually means working with multiple software platforms, spreadsheets, documents, etc. Various studies highlight that $33 \%$ of companies have more than ten HR systems. $47 \%$ of companies have HR software that is over seven years old [10]. According to Center for Effective 
Organizations and a study conducted by G\&A Partners, HR professionals spend about $73.2 \%$ of their time dealing with tedious administrative tasks [11]. But some of these HR operations could be automated with RPA-based solutions. But a successful implementation depends on several factors, including the RPA tool.

\section{B. RPA Tools}

Currently, there are many RPA tools available on the market, some of them free, others commercial, such as, Automation Anywhere, Blue Prism, EdgeVerve, Microsoft, NICE, UiPath, WorkFusion, etc.

RPA market solutions offer various capabilities, as presented in numerous studies and scientific papers, such as, for example, [12]. It should be noted that providers of robotic process automation platforms quickly and continuously update their offers to meet the developing needs of their clients. Thus, for example, various RPA providers announced further development of their RPA platforms, by incorporating new breakthroughs in artificial intelligence and machine learning. Given the multitude of existing RPA platforms that offer powerful functionality, as well as their continued development, choosing the best automation tool for an organization could be a real challenge.

\section{RELATED WORK}

Recently, because of the difficulties caused by the COVID19 pandemic, many companies have turned to robotic process automation solutions to help them overcome the challenges of the pandemic. Thus, they can "automate repetitive tasks across multiple business applications without altering existing infrastructure and systems” [13].

Many publications present the strengths and weaknesses of the RPA. However, the reviewed literature highlights a predominantly positive assessment of RPA, given that its strengths outweigh its weaknesses [14]. Adopting RPA in enterprises offers many benefits, including higher productivity, improved business efficiency and accuracy, data security, scalability, auditability, low printing and storage [13]. The promises of RPA for enterprises include easy implementation at a relatively low cost compared to other solutions.

Some authors consider that RPA is suitable for processes with high levels of i) standardization, ii) volume of transactions, iii) maturity, iv) approach to business rules ([13], [15], [16], [17]). Other authors recommend the RPA approach for standardized and repetitive processes that i) follow business rules, ii) take longer to complete, iii) are performed on a regular basis, and iv) require manual interaction with information systems [18]. Furthermore, many authors advocate RPA adoption for processes involving structured data, low variance and logic-driven procedures [19].

\section{Discussion}

The analysis of scientific and industry publications shows that RPA has been used moderately in the human resource departments of large enterprises and less in SMEs. A single enterprise can have hundreds or thousands of processes and sub-processes and the selection of those that could be automated with RPA is a difficult task.
We examined case studies that are related to the aforementioned research questions. Following an examination of these case studies, and an analysis of the academic and industry papers it can be highlighted that the processes suitable for RPA have several common features. Thus, the considered process should have clearly defined inputs and outputs. Workforce tasks that are rule-based, predictable, high-volume, time-consuming, repetitive, or prone to human error are good candidates for RPA.

Despite the vast potential of RPA technology, experts point out that it is not suitable everywhere. Thus, if a process is dynamic, requires creative thinking, deals with unexpected events or involves decision-making on a case-by-case basis, RPA can fail.

Various studies have shown that RPA projects failed initially in a proportion of $30 \%$ to $50 \%$ [20]. In order to determine which processes are suitable for RPA, an evaluation can be performed considering the criteria specified above.

As we can see, there are many opportunities to use RPA in human resource management. Below we present some HR processes in SMEs that can be automated with RPA, in response to the RQ2. Table I summarizes the HR use cases found in the literature and validated by some HR experts.

TABLE I. THE RPA OPPORTUNITY FOR HR DEPARTMENTS

\begin{tabular}{|c|c|c|c|}
\hline $\begin{array}{l}\text { HR Process to } \\
\text { be automated }\end{array}$ & Benefits & $\sum_{\infty}^{1}$ & 离 \\
\hline \multirow{10}{*}{$\begin{array}{l}\text { CV Screening } \\
\text { and recruitment }\end{array}$} & Facilitating employment & $\mathrm{X}$ & \\
\hline & Identifying the best candidates & $\mathrm{X}$ & \\
\hline & $\begin{array}{l}\text { Keep candidates informed with better, } \\
\text { automate communication }\end{array}$ & $\mathrm{X}$ & $\mathrm{X}$ \\
\hline & Faster cross verification of candidates' details & $\mathrm{X}$ & \\
\hline & $\begin{array}{l}\text { Automatic data concatenation from multiple } \\
\text { input sources }\end{array}$ & $\mathrm{X}$ & \\
\hline & Behavior reference check & $\mathrm{X}$ & \\
\hline & Criminal record checks & $\mathrm{X}$ & \\
\hline & Correct evaluation of candidates & $\mathrm{X}$ & $\mathrm{X}$ \\
\hline & Minimizing delays in hiring process & $\mathrm{X}$ & \\
\hline & Reduction in hiring costs & $\mathrm{X}$ & \\
\hline \multirow{4}{*}{ Onboarding } & Smooth onboarding process & & $\mathrm{X}$ \\
\hline & Data integration capacity & $\mathrm{X}$ & \\
\hline & Faster onboarding process & & $\mathrm{X}$ \\
\hline & Low-cost onboarding process & $\mathrm{X}$ & \\
\hline \multirow{3}{*}{$\begin{array}{l}\text { Employee } \\
\text { training }\end{array}$} & Accelerated employee skill acquisition & & $\mathrm{X}$ \\
\hline & $\begin{array}{l}\text { Better alignment of employee skills with the } \\
\text { organizational certification requirements }\end{array}$ & $\mathrm{X}$ & \\
\hline & Enhanced learning experience & & $\mathrm{X}$ \\
\hline \multirow{3}{*}{$\begin{array}{l}\text { Employee data } \\
\text { management }\end{array}$} & $\begin{array}{l}\text { Better management of data of current/former } \\
\text { employees etc. }\end{array}$ & $\mathrm{X}$ & \\
\hline & $\begin{array}{l}\text { Consistent actions across various systems/ } \\
\text { databases/departments }\end{array}$ & $\mathrm{X}$ & \\
\hline & Elimination of data entry errors & $\mathrm{X}$ & \\
\hline
\end{tabular}




\begin{tabular}{|c|c|c|c|}
\hline & Employee data protection & $\mathrm{X}$ & $\mathrm{X}$ \\
\hline & $\begin{array}{l}\text { Efficient allocation of office space in the } \\
\text { hybrid-work conditions }\end{array}$ & $\mathrm{X}$ & \\
\hline \multirow{3}{*}{$\begin{array}{l}\text { Tracking } \\
\text { attendance }\end{array}$} & Better calculation of employee salaries & & $\mathrm{X}$ \\
\hline & Workflow disruption prevention & $\mathrm{X}$ & \\
\hline & Accurate time records & $\mathrm{X}$ & \\
\hline \multirow{4}{*}{$\begin{array}{l}\text { Payroll } \\
\text { management }\end{array}$} & Avoiding delay in salary payments & & $\mathrm{X}$ \\
\hline & Lower payroll costs & $\mathrm{X}$ & \\
\hline & Reduced risk of multiple errors & $X$ & \\
\hline & Simplified payroll processing & $\mathrm{X}$ & \\
\hline \multirow{2}{*}{$\begin{array}{l}\text { Expense } \\
\text { management }\end{array}$} & Fast processing of expenses & & $\mathrm{X}$ \\
\hline & $\begin{array}{l}\text { Correlation of individual expenses with } \\
\text { company regulations and spending rules }\end{array}$ & $\mathrm{X}$ & \\
\hline \multirow{2}{*}{$\begin{array}{l}\text { Maintaining } \\
\text { compliance }\end{array}$} & $\begin{array}{l}\text { High accuracy of the maintaining compliance } \\
\text { process }\end{array}$ & $\mathrm{X}$ & \\
\hline & $\begin{array}{l}\text { Ensuring an error-free compliance } \\
\text { maintaining process }\end{array}$ & $\mathrm{X}$ & \\
\hline \multirow{2}{*}{$\begin{array}{l}\text { Exit } \\
\text { management }\end{array}$} & Exit process consistency & $\mathrm{X}$ & \\
\hline & Ensuring data privacy & $\mathrm{X}$ & \\
\hline \multirow{2}{*}{$\begin{array}{l}\text { Performance } \\
\text { management }\end{array}$} & $\begin{array}{l}\text { Faster data collection for the calculation of } \\
\text { performance indicators }\end{array}$ & $X$ & \\
\hline & $\begin{array}{l}\text { Automatic report generation/ updating/ } \\
\text { deletion }\end{array}$ & $\mathrm{X}$ & \\
\hline Qualitative & Quantitative & & \\
\hline
\end{tabular}

The general hire-to-retire (H2R) process integrates several disparate systems that often require swivel-chair work to migrate employee data between different systems. These processes are good candidates for RPA. Various studies have shown that recruitment is one of the most time-consuming key tasks for human resources. On average, recruiters spend $60 \%$ of their time for candidates sourcing and screening. Implementing an RPA-based solution can take over manual and repetitive work involved, such as filtering resumes, scheduling interviews, and simplifying the integration process.

RPA can significantly reduce inefficiencies on management reporting. Thus, an RPA-based solution can gather information from multiple sources and generate a report or save it into consolidated XLS.

The implementations we studied showed a significant decrease in process time and in errors, and a high potential for scalability.

Successful use cases will continue to emerge with the increasing development of RPA platforms and as more businesses expand their use of RPA, demonstrating the wide variety of issues that can be addressed.

Further on, we attempt to answer the RQ3 research question, presenting the benefits of adopting RPA. RPA has great value to offer for improving the human resource management system in small- and medium-sized enterprises. The benefits we mean are not only for the company, but also for employees. Thus, RPA-based solutions could offer SMEs important benefits, including increased productivity, improved accuracy, faster digital transformations, higher employee engagement, improved data security and quality, etc. Once an RPA layer has been added within an SME for HR processes, each individual employee can access and exploit it. RPA-based solutions could offer employees [13] a wide variety of benefits in laborious or tedious manual processes. We presented some of the qualitative and quantitative benefits identified following the analysis of the academic and industry literature.

After conducting the overview of various possibilities, the main conclusion is clear. RPA could play an important role to shift the HR functions to enter the digital era.

Next, based on literature research, we present some of the challenges that SMEs face in implementing RPA within HR departments (RQ4).

An important challenge for many SMEs is how to add automation to workflows. There can be a large number of processes and sub-processes within the HR departments of SMEs [21], so choosing which ones could be automated with RPA, as well as the order in which they can be automated can prove to be a big challenge.

Other challenges focus on human resource management, given that workforce need to adapt to new work, as RPA-based solutions can take on important parts of their daily tasks [22].

The challenges can also be considered from a legal viewpoint, analyzing, for example, questions such as "who has the control over the intellectual property robots handle and generate" and "who is responsible if the robot fails" [23]. Unfortunately, these two questions have not yet been answered.

\section{CASe Study}

Recruitment is one of the most promising use cases for adopting RPA-based solutions. Processes in this area involve a large volume of repetitive, time-consuming tasks that are still manually operated by human force. Thus, a significant amount of time is spent screening resumes and application forms submitted by candidates for open positions. Software robots can make this process considerably easier by gathering applications quickly and comparing all of the data to a list of precise job requirements. These requirements can be viewed as predetermined rules that influence the whole selection method while using RPA technology.

Candidates for a position in an SME scan their documents and send them to the SME for processing. Most of the times the documents sent by the candidates are in pdf format. These include diplomas, identity card, certificates, CV, letter of intent, etc. Some documents may have a format imposed by SME, in this case the centralization of information from several candidates is much simplified. For example, an SME can request the completion of important information in editable pdf files with PDF tagged order features. But, retrieving information from candidates' scanned documents is a tedious, repetitive, time-consuming process that involves a multitude of copy-paste activities and requires manual interaction with information systems.

To eliminate these disadvantages, we proposed an RPAbased solution. For this case study, we have opted for the 
UiPath platform. Thus, we developed a solution based on RPA enhanced with Optical Character Recognition (OCR) that can recognize and extract the information needed by the HR department from scanned documents and then enter it into the HR database. HR staff can run this solution on their laptop while handling more important activities. Then, the only operation to be performed by the HR department staff is to verify the veracity of the information entered in the system by the RPA solution.

The flow chart of this application is depicted in Fig. 2.

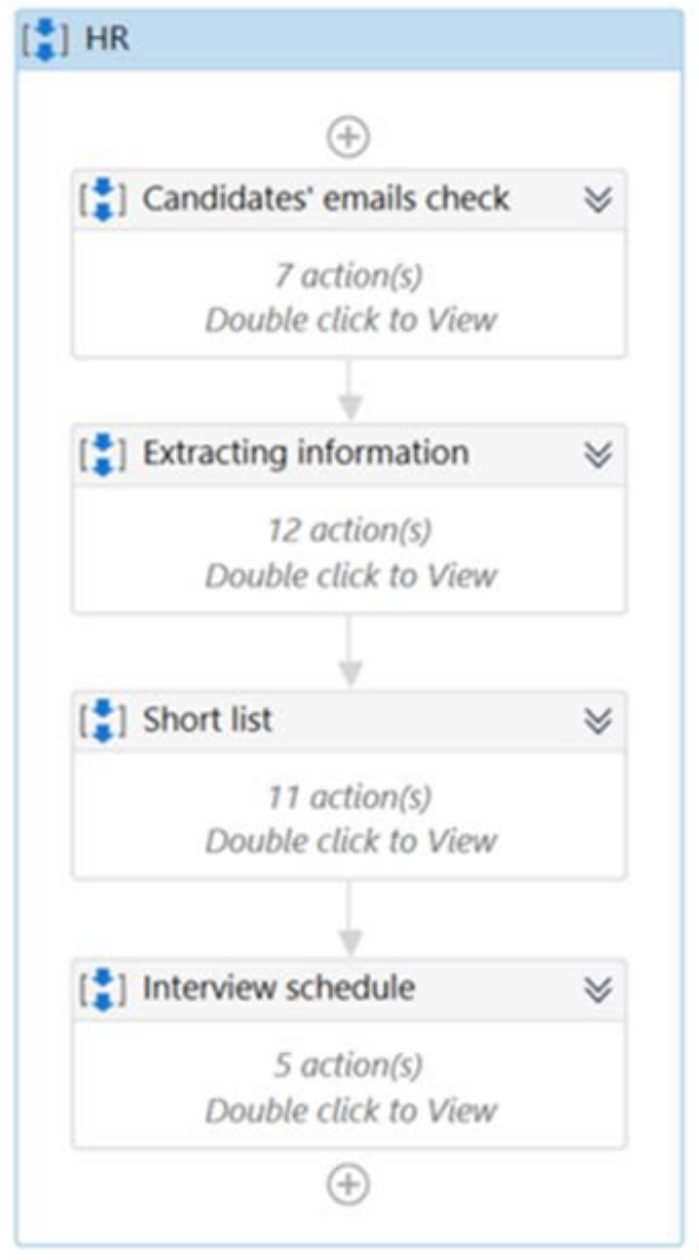

Fig. 2. The Flow Chart of the RPA-based Solution.

The proposed solution has been verified to ensure proper processing. Thus, we evaluated the implemented application with documents of different formats. In our experiments we used pdf files and image / picture files (JPEG, BMP, GIF, PNG, etc.). The extraction of information from the files sent by the candidates is one of the most significant difficulties to be handled. This problem becomes even more acute when the documents are edited in a language with special characters, as the Romanian language. Thus, the information on the applicants' documents may contain diacritics in Romanian. The correctness of the extracted information depends a lot on the OCR engine.
We have developed and tested several OCR engines available in UIPath, such as: Microsoft OCR, Google OCR, Abbyy OCR and other OCR software like: Convertio [24], Online OCR [25], Free Online OCR [26], i2OCR [27]. The best results were obtained using Convertio and Abbyy FineReader. Even if the developed application is in a beginning form, we consider that it is necessary to test as many OCR engines as possible and to select the one that provides the best recognition rate for Romanian characters.

One way to reduce the number of errors in extracting information from scanned files is to use OCR engines that are based on artificial intelligence. One such example is Abbyy FineReader, which has the latest OCR technology based on artificial intelligence and performs a variety of operations such as digitizing, retrieving, editing, protecting and sharing documents of various types in the same workflow [28].

A limitation of the research is the use of only one RPA platform (UIPath) for the case study. To create meaningful solutions for HR departments in SMEs, further research employing different RPA platforms is required.

The authors of this paper intend to continue research in the near future, considering further explorations of RPA in the field of human resource management in SMEs. Attention will be paid to combining solutions developed using RPA tools with solutions based on blockchain, Internet of Things, etc.

\section{CONCLUSION}

This paper aimed to explore the robotic process automation technology and to identify the promising application of RPA in human resource management within SMEs. In the near future, human resource departments that rely on manual, paper processes will need to re-examine their processes and consider whether RPA technology can benefit them. Through this paper, the authors want to support SMEs that aim to streamline the HR department by adopting RPA.

In the analysis of numerous publications and case studies, various authors' observations on how companies and business people are trying to reorganize the company's human resources for an accelerated economic recovery after being affected by the pandemic crisis, we tried to find answers to some of the basic problems of HR digitization, which would contribute to a profitable and sustainable business for SMEs. The adoption of RPA can bring important benefits in the HR departments within SMEs for laborious or tedious, repetitive, timeconsuming manual processes.

Through the case study implemented and presented, it was demonstrated that RPA can fulfill some of the basic preprocessing tasks undertaken by human resources departments in recruiting candidates.

The RPA platform used has an important role in the successful implementation of an RPA-based solution. There are various vendors on the market that offer RPA solutions. Choosing the best solution to meet the specific requirements of an SME's HR department is not an easy task, an in-depth analysis must be performed by taking into account several criteria. 
Thus, for example, a developer must consider the type of analyzed documents when implementing RPA-based solutions for managing candidates' documents (scanned or generated, with or without images, with or without tagged order features, etc.). The correctness of the extracted data is also dependent on the ability of OCR tools to recognize the specific characters of the language used in the elaboration of documents. In many cases, processing data that has been extracted incorrectly can be time-consuming.

Although some HR processes require human intervention to correct extraction errors, RPA can be considered a viable candidate for streamlining HR processes, which can lead to a rapid improvement in a business's overall value.

\section{ACKNOWLEDGMENT}

This research was funded by the project " $119722 /$ Centru pentru transferul de cunoștințe către întreprinderi din domeniul ICT-CENTRIC, Contract subsidiar 15569/01.09.2020, Platformă inteligentă pentru gestionarea resurselor umane - HR ASSISTant”, contract no. 5/AXA 1/1.2.3/G/13.06.2018, cod SMIS 2014+ 119722 (ID P_40_305).

\section{REFERENCES}

[1] Gregurec, M. Tomičić Furjan, and K. Tomičić-Pupek, "The impact of COVID-19 on sustainable business models in SMEs," Sustainability, vol. 13, no. 3, p. 1098, 2021.

[2] M. Chui, J. Manyika, and M. Miremadi, "Where machines could replace humans-and where they can't (yet),” 2016.

[3] F. Biscotti, V. Mehta, A. Villa, B. Bhullar, and C. Tornbohm, "Market share analysis: robotic process automation,” worldwide, 2019. Technical report, 2020.

[4] "Robotic Process Automation 101: a primer on automating IT-based tasks via bots imitating human behaviour - Reports \& Insights." https://transformainsights.com/research/reports/robotic-processautomation-technology-insight-report (accessed Nov. 19, 2021).

[5] "Digital HR | Deloitte | Human Capital,” Deloitte Danmark. https://www2.deloitte.com/dk/da/pages/human-capital/articles/digitalhr.html (accessed Sep. 20, 2021).

[6] "How bots, algorithms, and artificial intelligence are reshaping the future of corporate support functions | McKinsey.” Avaliable: https://www.mckinsey.com/business-functions/mckinsey-digital/ourinsights/how-bots-algorithms-and-artificial-intelligence-are-reshapingthe-future-of-corporate-support-functions?cid=other-eml-alt-mip-mckoth-

1811\&hlkid=a214ffef34fa4418b3bacbffe56be12d\&hctky=9653147\&hdp id=e7907fc7-7f94-4d63-a845-e2913105cc0d (accessed Nov. 27, 2021).

[7] PricewaterhouseCoopers, "2020 HR Technology Survey: Key findings," PwC. https://www.pwc.com/us/en/services/consulting/workforce-of-thefuture/library/hr-tech-survey.html (accessed Oct. 19, 2021).
[8] I. C. A. Group, "IEEE Guide for Terms and Concepts in Intelligent Process Automation.” IEEE New York, NY, 2017.

[9] R. Syed et al., "Robotic process automation: contemporary themes and challenges,” Computers in Industry, vol. 115, p. 103162, 2020.

[10] Josh Bersin, “15 Two Major Marketplace Issues,” Dec. 2014. [Online]. Available: https://www.slideshare.net/jbersin/ten-disruptions-in-hrtechnology-for-2015-ignore-at-your-peril/1215Two_Major_Marketplace_Issues1_Too

[11] “HR's Time-Consuming Toll On Your Company,” G\&A Partners, Jan. 15, 2015. https://www.gnapartners.com/resources/infographics/hrs-timeconsuming-toll-company (accessed Nov. 15, 2021).

[12] P. Hofmann, C. Samp, and N. Urbach, "Robotic process automation," Electronic Markets, vol. 30, no. 1, pp. 99-106, Mar. 2020.

[13] A. Leshob, A. Bourgouin, and L. Renard, "Towards a process analysis approach to adopt robotic process automation,” in 2018 IEEE 15th International Conference on e-Business Engineering (ICEBE), 2018, pp. 46-53.

[14] J. Hindel, L. M. Cabrera, and M. Stierle, "Robotic process automation: Hype or hope?," in Wirtschaftsinformatik (Zentrale Tracks), 2020, pp. $1750-1762$.

[15] M. Lacity and L. Willcocks, "Paper 16/01: Robotic Process Automation: The Next Transformation Lever for Shared Services.” Retrieved from The Outsourcing Unit, LSE: http://www. umsl. edu/ lacitym ..., 2016.

[16] M. Lacity, L. P. Willcocks, and A. Craig, "Robotic process automation at Telefonica O2,” 2015.

[17] L. Willcocks, M. Lacity, and A. Craig, "Robotic Process Automation at Xchanging,” The Outsourcing Unit Working Research Paper Series.

[18] P. Lowes, F. R. Cannata, S. Chitre, and J. Barkham, "The business leader's guide to robotic and intelligent automation," Deloitte Development LLC, 2017.

[19] C. Mendis, C. Silva, and N. Perera, "Moving ahead with Intelligent Automation,” 2016.

[20] “Can robots help your business be more human?" https://www.ey.com/en_gl/digital/can-robots-help-your-business-bemore-human (accessed Oct. 27, 2021).

[21] K. McCandless, "7 Essential Human Resources Processes for Small Businesses.” https://www.fool.com/the-blueprint/human-resourcesprocess/

[22] D. Choi, H. R'bigui, and C. Cho, "Robotic Process Automation Implementation Challenges," in International conference on smart computing and cyber security: strategic foresight, security challenges and innovation, 2020, pp. 297-304.

[23] C. Holder, V. Khurana, F. Harrison, and L. Jacobs, "Robotics and law: Key legal and regulatory implications of the robotics age (Part I of II)," Computer law \& security review, vol. 32, no. 3, pp. 383-402, 2016.

[24] Convertio - File Converter.

[25] Online OCR. [Online]. Available: https://www.onlineocr.net/.

[26] Free Online OCR. [Online]. Available: https://www.newocr.com/.

[27] i2OCR. [Online]. Available: http://www.i2ocr.com.

[28] ABBYY FineReader. [Online]. Available: https://pdf.abbyy.com/. 